\title{
Drei Briefe Hermann Brochs an Oscar Oeser Mitgeteilt von
}

\section{GERHARD SCHULZ}

Hermann Broch an Oscar Oeser 1 7. Juni 1939

H.B.dzt. 3171 Washington Boulevard, Cleveland Heights (Ohio), 7.6.39

Lieber Oskar,

haben Sie Dank für Ihre beiden Briefe; den ersten habe ich mit einer Karte nach Chicago beantwortet, die Sie vielleicht noch, vielleicht nicht mehr erhalten haben, aber ohnehin nur eine Adressenangabe war. ${ }^{2}$

Anbei nun der Entwurf zur Hervorbringung von Massenwahn. Sie werden meine New Havener und New Yorker Lehrjahre darin wiedererkennen. $\mathrm{Ob}$ sie mit der Fundierung im Werttheoretischen einverstanden sein werden, weiss ich nicht: ich fühlte mich zu dieser Fundierung des Modells gedrängt, nicht nur, weil ich über die erkenntnistheoretische und logische Stellung des Wertbegriffes schon viel nachgedacht habe, sondern noch weit mehr, weil man zu jedem Arbeitsgebiet - besonders wenn es quasi erstmalig umrissen werden soll - eine übergeordnete Bestimmungssphäre benötigt; anders als mithilfe eines derartigen "kleinsten gemeinsamen Vielfachen" kann ich mir die Züsammenfassung von so weitverzweigten Wissenselementen überhaupt nicht vorstellen.

Meine Fragen an Sie:

1.) Sind Sie mit dem Entwurf einverstanden oder welche Abänderungen würden Sie für wünschenswert erachten?

2.) Sind sie bereit einem Gründungskommitée für das Institut beizutreten?

3.) Haben Sie Ihrerseits Vorschläge für die Liste der Kommitéemitglieder?

4.) Möchten Sie mit einem kurzen Beitrag an dem Sammelwerk mitarbeiten, das zur Aufstellung eines detaillierteren Forschungsprogramms von der American Guild for German Cultural Freedom, oder richtiger mit deren finanzieller Beihilfe herausgegeben werden soll? 
Natürlich werden Sie wenig Zeit haben, und Ihre geldgierige Camparbeit, zu der ich Ihnen aber doch gratulieren muss und die ich für einen richtigen und überdies zukunftsträchtigen Entschluss halte, wird Ihr Zeitkonto unter Null bringen, d. h. Sie werden mit negativen Zeitbeträgen arbeiten müssen, und dies ist an und für sich ein Kunststück. Doch da es mit einem wienerischen Ausdruck heisst, dass "einem Geschwollenen nichts mehr schadet", so hoffe ich sehr, dass Sie zur Ueberbelastung auch noch die Ueber-Ueberbelastung werden addieren können. Sie haben das Unglück, für diese Sache schlechthin prädisponiert zu sein, und bei aller gebotenen Skepsis gegen die Sache (nicht gegen Sie) meine ich, dass daraus noch etwas sehr Anständiges erwachsen könnte.

Schreiben Sie bald ein Wort. Bis zum 16. oder 17. bleibe ich hier, (Adresse oben), ab 20. Juni bis 6. August bin ich in "Yaddo", Saratoga Springs (N.Y.) und ab 15. August werde ich für 4 Wochen im Hause Einsteins in Princeton sein, ${ }^{3}$ über dessen Einladung ich mich begreiflicherweise besonders gefreut habe. Und da Princeton nahe genug von N.Y. ist, kann ich Sie ab 6. August, soferne Sie bis dahin schon wieder im Osten sein sollten, jederzeit sehen.

Inzwischen alle guten Wünsche, in Herzlichkeit Ihr

[handschriftlich] Hermalın Broch

Ich habe meinerseits auch nichts aus St. Andrews gehört, nicht einmal von dem schreibfreudigen Stewart. ${ }^{4}$ Die Leute scheinen dort eine rätselhafte, wenn auch verständliche Schreibkrankheit zu haben.

Hermann Broch an Oscar Oeser 14. Juli 1939

Hermann Broch, "Yaddo",

Saratoga Springs (N.Y.)

14.7.39

Lieber Oskar,

eine zeitlang glaubte man, dass Sie zu den Sitten der Kulturvölker zurückgekehrt seien; nun zeigt es sich, dass Sie unrettbar in der St. Andrewerei verbleiben, wo bekanntlich Brief-Beantworten als Sünde gilt.

Nun weiss ich, dass Sie ebenso arbeitsüberlastet sind, wie ich es bin, und dass sogar eine Postkarte manchmal als unübersteigbares Hindernis erscheint. Aber trotzdem hätte ich gerne gewusst, ob Sie meinen Text bekommen haben oder nicht. Weiters möchte ich wissen, wo Sie sich befinden, etc.

$\mathrm{Zu}$ meinem Exposée habe ich zu berichten, dass ich es vorderhand bloss ein paar Analytikern und den Verbindungsleuten zu den in Aussicht 
genommenen Geldgebern gezeigt habe. Weiters habe ich es Einstein geschickt, der sich dafür interessiert und mich nach Princeton eingeladen hat: von $\mathrm{ihm}$ ist die erfreulichste Zustimmung gekommen (-für mich sehr überraschend --); er will seinen Einfluss bei der Finanzierung zur Verfügung stellen etc. Allerdings ist er gegen Kollektiv-Institute; in Ueberschätzung meiner Person, meint er, dass ich die Forschung allein mit Hilfskräften vorzunehmen hätte. Ebensogut könnte ich eine chirurgische Klinik leiten. Aber da wird er schon Vernunft annehmen.

Mein Modell zur Massenwahnerzeugung bedarf noch einiger Rektifikationen. Insbesondere kann -- wie ich aus der Diskussion gesehen habe - aus dem jetzigen Text missverständlich der Schluss gezogen werden, ich leite alles Wertstreben aus der Angst ab. Das stimmt selbstverständlich nicht, war auch von mir nicht so intendiert (da ich ja nicht psychologisch, sondern erkenntnistheoretisch basiere), und wenn man sich auch hiezu auf das grosse Beispiel Kierkegaards berufen könnte, ich tue es nicht, sondern werde dies noch klarstellen. Dann erhalten Sie die endgültige Fassung. Praktisch geht es nun darum, ein Einladungskommitée zusammen zu stellen. Und da kann ich Sie nicht auslassen; da müssen Sie mithelfen, zumindest durch Vorschläge, oder Sie müssen eben selber Mitglied werden. Durch Verstecken werden Sie sich nicht entziehen.

Der Sicherheit halber schicke ich den Brief in Doppelparie ab, einmal durch meinen Freund Bergmann ${ }^{5}$, der sich jetzt mit Lewin 6 in Berkeley befindet und Ihre Adresse dort ausfindig machen soll, das andermal durch das Institut for Human Relation?.

Ich bin bis Anfang August hier (- nahezu ausschliesslich mit dem Vergil beschäftigt -) und $a b$ 15. August bei Einstein in Princeton (N.J.), 112 Mercer Street. Zwischendurch ein paar Tage in Boston und New York. Ich rechne bestimmt darauf, Sie zu sehen. Von Princeton kann ich ja jederzeit nach N.Y. kommen. Ausserdem hatten Sie, so viel ich mich erinnere, die Absicht, nach Princeton zu fahren. Inzwischen aber schreiben Sie doch jene Postkarte.

Alles Herzliche Ihres

[handschriftlich] HB. 
"Yaddo", Saratoga Springs, N.Y., 30.7.39

Geht der Dichter dran, die Massen

Wissenschaftlich zu erfassen,

Muss er sich's gefallen lassen,

Dass zum Wissenschaftserlöser

Reimend wird der Oskar Oeser.

Leider ist es, lieber Oskar, nicht auf Englisch geglückt, denn auf Oeser will sich Poet nicht reimen lassen, sondern bloss Proser, und so habe ich es aufgegeben. Aber Bild und Gedicht habe ich begeistert an Ruth Norden 8 weitergeschickt. Und im übrigen kann ich Sie ob Ihrer Ueberarbeitung nicht bemitleiden, denn wenn Sie Ihre Zeit dransetzen, ein deutscher Klassiker zu werden, verdienen Sie es nicht besser.

So weit die wichtigen Angelegenheiten, nun die unwichtigen:

Dass Sie ins foundation committee einzutreten bereit sind, ist zweifelsohne von aller Bedeutung für die ganze Angelegenheit; allerdings sollen Sie sich vor Augen halten, dass Sie von Zeit einen Brief vermittels einer Postkarte werden beantworten müssen, und dass an derselben alles scheitern kann.

Bevor jedoch ein solches Kommitee gebildet werden darf, erscheint es mir notwendig, sich mit den bereits in Gange befindlichen Bestrebungen ähnlicher Natur zu befassen: in Colorado besteht eine Gruppe unter der Leitung von J. Krechevsky9 "Society for the Psychological Study of Social Issues", und in Washington soll Harold Lasswell10 (- von Institut for Human Relations! -) eine Art Forschungsinstitut u.z. mit stark psychoanalytischen Einschlag betreiben. So bald ich das Material habe, welches der Analytiker Spitz ${ }^{11}$ zur Vervielfältigung, resp. Uebersetzung übernommen hat, will ich mich mit den beiden Stellen in Verbindung setzen. Sollten Sie jedoch Ihrerseits dorthin direkte Beziehungen haben - und dies scheint mir bei Lasswell durchaus möglich zu sein -, so schreiben Sie mir bitte bereits eine jener Postkarten, u. z. ob ich mich auf Sie berufen darf. Wissen Sie vielleicht schon etwas über den Bestand jenes Institutes in Washington? ich halte es nicht für ausgeschlossen, dass Ihnen inzwischen davon bereits etwas zu Ohren gekommen sei.

Ob Ihrer Deteilkritik bin ich geehrt, erfreut und gerührt. Aber ich kann vorderhand noch nicht darauf eingehen, weil ich kein einziges Exemplar meines Textes zur Hand habe: meine Kopien sind alle ausgeschickt, und die neuen lassen eben auf sich warten. Nur zum Einwand Nr. 1 kann ich sofort Antwort geben: natürlich sind aus Modellbildungen bereits Wissenschaften entstanden: Marx hat ein nationalökonomisches, Freud ein psychisches Modell aufgestellt. (Ueber die definitorischen Unter- 
scheidungen zwischen Theorie, Hypothese und Modell wäre natürlich einiges zu sagen, doch erscheint mir eben der Ausdruck Modell am geeignetsten, er erscheint mir auch richtiger als etwa "Hypothesensystem".) Sohin beschränke ich mich für heute darauf, Ihnen ein Einlageblatt $4 \mathrm{~A}$, zu meinem Text zu senden; es ist der Ersatz für den letzten Absatz auf Seite 4 und die ersten 8 Zeilen auf Seite 5.

Und im übrigen kann ich nur die Formel wiederholen, die der Jude aus Wien seinen amerikanischen Verwandten telegraphiert hat: "Seid besorgt, Brief folgt."

Betr. Besorgnis: haben Sie Nachricht über Willas Befinden??! Alles Herzliche Ihres

[handschriftlich] Hermann

[handschriftlicher Zusatz am Rand]:

Dank für die Briefnachsendungen! Ich bleibe bis 10. hier. Und ab 15., wie gesagt: 112 Mercer Street, Princeton, N.J.

Von den hier mitgeteilten drei Briefen Hermann Brochs hörte ich zuerst in kollegialem Gespräch. Beiläufig nur hatte Oscar Oeser - bis 1969 Professor für Psychologie an der University of Melbourne und seitdem Emeritus Professor dieser Universität - seine Bekanntschaft mit dem deutschen Schriftsteller erwähnt, auf die Nachfrage hin dann aber längst beiseitegelegte Dokumente ans Licht gebracht. Es waren die hier wiedergegebenen drei Briefe Brochs und dazu eine Kopie von dessen Vorschlag zur Gründung eines Institutes für politische Psychologie und zum Studium von Massenwahnerscheinungen 12, worauf Broch im ersten Brief - vom 7. Juni 1939 - mit der Abbreviatur "Entwurf zur Hervorbringung von Massenwahn" Bezug nimmt, sowie der Durchschlag von Oesers Antwortbrief und Kommentar vom 24. Juli 1939. Im Brief vom 14. Juli hatte Broch eine solche Antwort angemahnt, im dritten Brief vom 30. Juli bedankt er sich dann dafür. Frühere Korrespondenz zwischen Broch und Oeser, auf die Broch am 7. Juni Bezug nimmt, ist leider verlorengegangen, ebenso ein kleines deutsches Gedicht Oesers,ein Akrostichon auf den Namen B-R-O-C-H, das er seinem Brief vom 24. Juli beigelegt hatte und auf das Broch dann mit dem eigenen Gedicht am Anfang des Briefes vom 30. Juli anspielt.

Broch und Oeser hatten einander an der Universität St. Andrews in Schottland kennengelernt. Broch war Anfang August 1938 nach seiner Emigration aus Österreich dorthin gekommen, eingeladen von dem Schriftstellerehepaar Edwin und Willa Muir, den englischen Ubersetzern von Brochs Romanen Die Schlafwandler und Die unbekannte Größe. Broch hatte seit 1931 mit ihnen in enger brieflicher und persönlicher Verbindung gestanden; seine Briefe an Willa Muir schwollen, wie Eric Herd schreibt, "zu einem ästhetischen Kommentar über sein Werk an, worin die großen Probleme der Kunst, wie sie Broch zu dieser 
Zeit beschäftigten, behandelt werden."13 Oeser, damals Dozent für Psychologie und Leiter des psychologischen Instituts in St. Andrews, gehörte zum Freundeskreis der Muirs, der 'St. Andrewerei', wie Broch das im Brief vom 14. Juli nennt. Anfang Oktober 1938 ging Broch dann jedoch nach Amerika. Er fühle den Blutsauger Hitler schon im Nacken, habe er damals nach Oscar Oesers Erinnerungen geäußert und hinzugefügt: "Ich muß weiter - ich kann und will den Krieg nicht mitmachen".

Im Dezember 1938 reiste auch Oeser nach den Vereinigten Staaten; er hatte eine 'Leverhulme Travelling Scholarship' erhalten, die ihn nach Harvard und, $a b$ März 1939, auch nach Yale führte. In seinem ersten Brief, den er aus Amerika an Willa Muir schrieb (3. 12. 1938), hatte Broch sogleich gefragt: "Was ist mit Oeser? Oeser Jedenfalls bitte um seine amerikanische Adresse."14 Es war nicht nur freundschaftliche Anteilnahme, die Broch zu dieser Bitte veranlaßte, sondern zugleich auch fachlich-wissenschaftliches Interesse, denn Oeser hatte in St. Andrews eines der ersten 'Research Teams' zusammengestellt, das sich mit sozialpsychologischen Problemen befaßte, und zwar mit der Arbeitslosigkeit in Schottland, wobei besonders die Interrelation von psychologischen, psychiatrischen, soziologischen und volkswirtschaftlichen Aspekten untersucht wurde. Bei Broch wiederum war mit der Arbeit an seinem Bergroman (seit 1935) und vor allem mit seinen ganz persönlichen Erfahrungen der Hysterie des Nationalsozialismus die Beschäftigung mit Fragen der Massenpsychologie stark gewachsen. Der Versuch zur wissenschaftlichen Durchdringung, Darstellung und Bewältigung solcher Phänomene im Dienste der Humanität trat nun, nach der Emigration, immer mehr in den Vordergrund. Der Bergroman war 1937 als Fragment liegengeblieben. Neben der Arbeit am Tod des Vergil, die im gleichen Jahr begonnen wurde, entfaltete Broch in der folgenden Zeit seine psychologischen Studien in großer Breite und Tiefe.

Oeser war für Broch als fachpsychologischer Berater von besonderer Bedeutung. Von den Methoden seiner sozialpsychologischen Forschung war für die Massenpsychologie viel zu lernen; Oeser kannte sich zudem in der angelsächsischen Forschung aus und sprach - er hatte in Marburg promoviert - fließend deutsch. Eine Verbindung der beiden in Amerika muß bald wieder zustandegekommen sein. Oscar Oeser selbst erinnert sich, daß ihn Broch vom 17. bis 20. April 1939 im Davenport College in Yale besuchte: "Er war mitten im Schreiben des Tod des Vergil. Kaum hatten wir uns begrüßt, als er seine Schreibmaschine aufstellte und - als ob er schon seit Wochen hier zu Hause wäre weitertippte." Broch gab damals Oeser eine Kopie des ersten Kapitels. Vom Eindruck überwältigt berichtete dieser in einem Brief nach St. Andrews: "I've read part of Hermann's Death of Virgil. One sentence ran over 3 pages. But don't smile - it was lucid, luminous, evocative prose. I'm astounded and almost incredulous as to what he has done to and with German prose! I should hate to have to try and translate it into English: it would be as difficult as trying to translate lyric poetry." Auf die Begegnung der beiden folgt dann der hier 
vorgelegte Briefwechsel.

Brochs Briefe geben einen deutlicheren Einblick in das erste Jahr seines amerikanischen Exils, als man ihn bisher hatte. Das Wanderleben des nahezu Mittellosen wird sichtbar. Anfang 1939 hatte ihm Henry Seidel Canby, Herausgeber der Saturday Review, einige Wochen Asyl in seinem Landhaus in Killingworth, Connecticut, gegeben. Ein Stipendium ermöglichte Broch, die Zeit vom 20. Juni bis zum 6. August - nicht mehrere Monate, wie man bisher annahm - in der Schriftstellerkolonie 'Yaddo', Saratoga Springs, zuzubringen. Dazwischen lag - unter anderem - der Besuch bei Oeser, und darauf folgte der Aufenthalt bei Albert Einstein in Princeton, wo sich Oeser und Broch noch einmal kurz trafen. Die Verbindung zwischen Emigration und Migration ist offensichtlich nicht nur eine philologische.

Tiefer noch als alle äußere Misere mußte jedoch auf die Dauer jene innere Fremdheit wirken, in der sich ein so stark aus Eigenem lebender deutscher Schriftsteller gegenüber den amerikanischen akademischen und politischen Institutionen befand, auf die er doch Einfluß ausüben wollte und von denen er Hilfe erwartete. Bei alledem war es ein Glück für Broch, an eine Gesellschaft geraten zu sein, die von einer in der angelsächsischen Tradition begründeten hohen Toleranzgrenze bestimmt wurde. Auch darüber und darüber vor allem gibt der Briefwechsel mit Oeser Auskunft.

Im Mittelpunkt der Beziehungen beider Männer steht Brochs Vorschlag zur Gründung eines Forschungsinstitutes für politische Psychologie und zum Studium von Massenwahnerscheinungen. Der Inhalt ist später in seine massenpsychologischen Arbeiten eingegangen 15, aber im Jahre 1939 war es ihm weniger darum zu tun, wissenschaftliche Erkenntnisse an und für sich zu verbreiten, als vielmehr die Öffentlichkeit für ein Projekt zu interessieren, das ihm aus doppeltem Grunde wichtig war. Die Verheerungen des Faschismus hatte er am eigenen Leibe erfahren, und es konnte für ihn nichts Bedeutenderes geben, als der Ausbreitung einer solchen alle Kultur und Menschlichkeit vernichtenden Seuche Einhalt zu gebieten, sie in ihren Ursachen und Keimen zu erkennen und von dorther auszurotten. Zugleich aber durfte er auch hoffen, in einem auf seinen Vorschlag hin gegründeten Forschungsinstitut einen festen Platz und damit materielle Sicherung zu erhalten. Brochs Vorschlag läuft darauf hinaus, $\mathrm{da} \beta$ in einem solchen Institut Einzelwissenschaften wie Theologie, Philosophie, Psychologie, Pädagogik und Kriminologie zu interdisziplinärer Zusammenarbeit gebracht werden mit dem gemeinsamen Ziel, massenpsychologische Manifestationen wie eben den europäischen Faschismus in seinen Motivationen und Mitteln zu erforschen. Das Ergebnis sollte dann sein, die "irrationalen Momente aus der Sphäre des blossen Instinktes zu heben, sie rational erfassbar zu machen und eben hiedurch in den Dienst des humanen Fortschrittes zu stellen." Das werde die "neue politische Aufgabe der Wissenschaft".

Zu diesem Zwecke stellt Broch ein 'theoretisches Modell' auf, das als Arbeitshypothese den Ausgangspunkt für alle weitere Diskussion bieten soll und den 
eigentlichen Inhalt seines Vorschlags darstellt. Für Broch ist die in existentieller Unsicherheit des Menschen begründete Angst der Ausgang aller massenpsychologischen Reaktionen, wobei er den Begriff Existenz durchaus nicht nur ontologisch, sondern auch materiell-ökonomisch versteht, also die in Wirtschaftskrisen, Arbeitslosigkeit und Inflation bedingte Existenzangst mit einschließt, ja sie sogar in den Vordergrund rückt. Angstbefreiung nun ist das eigentliche Ziel aller Kultur, eine Angstbefreiung, die schließlich in der Form von Ekstase erlebt werde. "Kultur", schreibt er, "ist rationale Regelung und Kontrolle irrationaler Bedürfnisse", und das Bedürfnis nach Befreiung aus der Angst rangiert an erster Stelle. Dabei gebe es allerdings zwei Möglichkeiten, nämlich den einer positiv zu verstehenden Irrationalbereicherung durch Religion, Kunst und die ethischen Lebensformen einer Gemeinschaft, andererseits aber auch den der 'Rationalverarmung', eine durch 'pseudorationale Begründungen' und 'eine Art Scheinethik' veranlaßte 'unethische Auslebung der unkontrollierten Triebe'. Hier eben entstehe Massenwahn. Charakteristische Erscheinungsformen davon seien 'Intellektualverachtung', die Vernachlässigung des gedruckten Wortes zugunsten des 'Bildhaften, als Zeitungsillustration und auf der Kinoleinwand' - die Bildröhre des Fernsehens war damals noch unbekannt - der 'zahlenmässige Ausdruck als messbarer Rekord oder als messbarer Gelderfolg' und schließlich die Angstbefreiung durch 'billige Kollektivekstasen im Kino' und 'auf den Sportplätzen'. Die 'Bildsehnsucht' stelle im Grunde eine 'Symbolsehnsucht' des 'panikbedrohten Menschen' dar, und so sei es letzten Endes nur folgerichtig, wenn man seine Angst auf wehrlose Minderheiten projiziere mit dem 'archaisch-infantilen' Wunsch 'nach konkret-physischer Vernichtung dieses lebenden Angst-Symbols'.

Auf diese Weise hat Hermann Broch versucht, auch mit der ihn unmittelbar betreffenden Tatsache des faschistischen Antisemitismus fertigzuwerden; dadurch, daß er das Phänomen in einem Begriffssystem unterbrachte, also den Erreger isolierte, hoffte er, zu dessen Vernichtung und damit zur Heilung von der Krankheit beizutragen.

Für den Broch-Leser werden Entwicklungslinien von den Schlafwandlern (1931-33) her deutlich. Die Rolle von Irrationalität und Werten in der modernen Gesellschaft waren bereits dort ein dominierendes Thema gewesen, während dann die massenpsychologischen Theorien vor allem aus der literarischen Praxis des Bergromans hervorwuchsen. Manches an Brochs Analyse ist von frappierender Richtigkeit geblieben, denn der Welt hat es bis heute an Massenhypnosen durch Verführer und an Verführungen gewiß nicht gemangelt. Anderes in Brochs 'theoretischem Modell' erscheint hauptsächlich als Spekulation eines gebildeten Schriftstellers, der religiösen und philosophischen Traditionen eng verbunden war, dessen eigene Theorien aber im Grunde nur durch das Kunstwerk legitimiert werden, aus dem sie hervorgehen und zu dem sie wieder zurückführen. Wie immer es aber mit dem Verhältnis zwischen Theorie und ästhetischer Gestaltung im Werk Hermann Brochs bestellt sein mag - sicher ist und war auch damals schon, daß er mit seinem Vorschlag zur Gründung eines 
Forschungsinstitutes kaum das erreichen konnte, was er erreichen wollte: staatliche und private Mäzene für ein solches Unternehmen zu interessieren.

Der Entwurf in seiner ganzen deutschen Theorieschwere hatte kaum Aussicht gehört zu werden, wenn es um pragmatische Ziele ging. Es sind jedoch nicht nur die Differenzen zwischen Philosoph und Geschäftsmann oder geisteswissenschaftlicher Spekulation und naturwissenschaftlicher Empirie, die hier manifest werden, sondern auch die prinzipielleren zwischen deutschem theorieorientiertem Denken und angelsächsischer fallorientierter Pragmatik. Es sind Unterschiede, die erst begriffen werden müssen, ehe eine Brücke zwischen ihnen möglich ist. Es ist das besondere Verdienst Oscar Oesers, Hermann Broch dieses Problem nahegebracht zu haben.

Wie schon erwähnt, beantwortete Oscar Oeser die Fragen Brochs in einem Brief vom 24. Juli 1939. Geschrieben wurde er während Oesers anstrengender Tätigkeit in 'work camps' für Studenten, d. h. in Lagern, die von der 'Central Quaker Organisation' in verschiedenen amerikanischen Notstandsgebieten ein gerichtet worden waren und in denen es um die Verbindung von sozialpsychologischen Studien mit praktischer Hilfeleistung ging. Er wolle gern, teilt er darin Broch mit, dem Gründungskomitee angehören, "if the committee as a whole has serious scientific standing"; er nennt Broch die Namen einiger Psychologen und Soziologen, die dafür zu interessieren wären - "a philosopher would in all probability be a mere nuisance, at any rate during the initial stages of planning and carrying out empirical research" - und er muß schließlich die Mitarbeit an einem Sammelwerk ablehnen, "because I am already almost impossibly involved in Sammelworking." In Oesers Kommentar zu den Brochschen Begriffen der Irrationalbereicherung und Rationalverarmung kommt dann der eigentliche Punkt seiner Kritik, die hier vollständig wiedergegeben werden soll:

Though I like the ideas of 'impoverishment and enrichment of the ratio', I feel that these two paragraphs are so involved, dogmatic and hypothetical that they ought to be cut or rewritten and expanded in less philosophical and speculative terminology. It does not pay to give the impression that the problems which are to be investigated have already been solved.

What evidence is there that values are always based on release from Angst! That all parts of the world which have not been incorporated by the self are regarded as symbols of death? Again, you may be right; but it seems to me unwise to state in a memorandum, designed to solicit funds, $a$ priori what may be the result of prolonged research, a posteriori.

Throughout, wherever you state something dogmatically, as though it were a confirmed result of empirical research, I would state it in the form of a hypothesis to be tested, or in the form of a question: "Is it possible to say that ..."; "let us make the hypothesis that ..."; "if we assume 
that . . ., then ...", and so on. In the first place, that seems to me the more adequate scientific procedure; pedestrian, perhaps, but necessary (though not sufficient). In the second place, empirical scientists are simply irritated by dogmatic philosophical pronunciamientos, and would therefore not give the matter the attention it deserves. (I have in mind English and American scientists. Germans are more accustomed to speculative metaphysics).

Darüber hinaus enthält Oesers Brief noch einige Bemerkungen zu einzelnen Stellen von Brochs Vorschlag: zum Begriff eines 'theoretischen Modells', zur 'Massenseele' und zu einigen praktischen Fragen möglicher Arbeitsorganisation. Sein entscheidender Rat ist jedoch in den oben zitierten Abschnitten enthalten. Vielleicht ist es nötig hinzuzufügen, daß ein solcher Rat nur möglich ist, wenn der Ratgeber selbst genügend Distanz zur eigenen empirischen und genügend Verständnis für die andere, spekulative Position hat, so daß er sie beide überblicken kann. Daß Broch dankbar für Oesers Mühen war, ohne wohl den Kern der Kritik recht verstanden zu haben oder ihn bei seinen Voraussetzungen überhaupt verstehen zu können, mag man aus seiner Antwort vom 30. Juli 1939 ablesen. Interessant ist es zu beobachten, daß sich die jüngste kritische Auseinandersetzung mit Brochs Massenwahntheorien tatsächlich auf der Linie von Oesers Argumenten bewegt. 16

$\mathrm{Zu}$ weiterer Kommunikation zwischen Oeser und Broch ist es dann nicht mehr gekommen. Brochs Plan wurde nicht verwirklicht, obwohl er selbst immerhin von 1942 bis 1944 eine Anstellung als Forschungsassistent an der Universität Princeton erhielt. Oeser kehrte bei Kriegsausbruch nach St. Andrews zurück.

\section{Anmerkungen}

An erster Stelle möchte ich Oscar Oeser danken, daß er mir seine Korrespondenz mit Hermann Broch zugänglich machte, mir die Erlaubnis gab, "to give my fragments of the Brochian Nachlass a decent academic burial", und daß er mich schließlich dabei mit vielfältigen Auskünften freundlich unterstützte. Die Briefe und das Manuskript des Vorschlags befinden sich jetzt im Besitz des Deutschen Literaturarchivs Marbach; seinem Direktor, Prof. Dr. Bernhard Zeller, danke ich für die Zustimmung zur Veröffentlichung. Auch die Erben Hermann Brochs, Frau Annemarie Broch (St. Cyr-sur-mer) und Herr H.F. Broch de Rothermann (New York), waren dankenswerterweise mit dem Abdruck der Broch-Briefe einverstanden. Dr. Christa Sammons, Kurator des Broch-Archivs in der Yale University Library, wo Oesers Brief aufbewahrt wird, hat für mich nach dem kleinen Oeserschen Akrostichon auf den Namen Broch gesucht. 
Die Briefe Brochs sowie auch der Brief Oesers sind Typoskripte auf Papier in der amerikanischen Standardgröße und mit sehr geringfügigen handschriftlichen Korrekturen. Die Wiedergabe erfolgte diplomatisch getreu; auch die wenigen orthographischen Unsicherheiten ("Kommitée" - dt. Komitée, engl. committee) oder Schreibfehler ("Deteilkritik") blieben unkorrigiert.

1 Oscar Oeser, geb. 1904 in Pretoria (Südafrika), Dr. phil. (Marburg) und Ph.D. (Cambridge), 1933 - 1946 Dozent für Psychologie und Leiter der Abteilung für experimentelle Psychologie an der Universität St. Andrews, 1946 - 1969 Professor für Psychologie an der Universität Melbourne, seit 1970 Emeritus Professor.

2 Diese Korrespondenz ist verlorengegangen (vgl. Kommentar).

3 Gemeint ist Albert Einstein, bei dem sich Broch dann mehrere Wochen im August/September 1939 aufhielt.

4 . Bill Stewart, Dozent für Französisch in St. Andrews. Broch erwähnt ihn auch in seinem Brief an Willa Muir vom 3.12. 1938 (vgl. Gesammelte Werke, Bd. 10, Zürich 1961, S. 368).

5 Gustav Bergmann, geb. 1906, Psychologe und Mathematiker, verließ Österreich 1938 und wurde 1940 Professor für Psychologie und Philosophie in Iowa.

6 Kurt Lewin, 1890 - 1947, Psychologe, Vertreter der Berliner Schule der Gestaltpsychologie, emigrierte bald nach 1933 und war zuletzt am Massachusetts Institute of Technology in Cambridge (Mass.) tätig.

7 Institute for Human Relations in Yale, wo Oscar Oeser arbeitete. Arbeitsbereiche des Instituts waren Anthropologie, Psychologie und Psychiatrie.

8 Ruth Norden, eine Bekannte Brochs in Chicago.

9 Isadore Krechevsky, geb. 1909, Psychologe, der seit 1931 an verschiedenen amerikanischen Universitäten vor allem auf dem Gebiet der Verhaltenspsychologie arbeitete. Er publizierte später unter dem Namen Krech.

10 Harold Lasswell, geb. 1902, Politologe, der von 1938 - 1939 an der Washington School of Psychiatry arbeitete, seit 1946 dann als Professor für Politologie in Yale.

11 René Spitz, geb. 1887, Psychoanalytiker, den Broch von Wien her kannte; er erwähnt ihn bereits am 29. 7. 1931 in einem Brief an Dr. D. Brody (vgl. Gesammelte Werke, Bd. 8, Zürich 1957, S. 58). Spitz emigrierte 1938 nach den Vereinigten Staaten und lebte zunächst in New York, seit 1956 an der University of Colorado (Denver).

12 Der Vorschlag ist in mehreren Exemplaren erhalten, u. a. im Broch-Archiv der Yale University' (vgl. Christa Sammons, "Hermann Broch Archive. 
Yale University Library", Modern Austrian Literature 5 (1972), Nr. 3/4, S. 39, Titel Nr. 68, 13). Veröffentlicht wurde er zuerst in: Hermann Broch, Zur Universitätsreform. Hrsg. und mit einem Nachwort versehen von Götz Wienold. Frankfurt a.M. 1969 (edition suhrkamp 301), S. 78 - 114. Jetzt auch in: Hermann Broch, Massenwahntheorie. Beiträge $z u$ einer Psychologie der Politik. (Kommentierte Werkausgabe, hrsg. von Paul Michael Lützeler, Bd. 12). Frankfurt a.M. 1979, S. 11 - 42. Der Abdruck dort ist mit folgender Anmerkung versehen:

"Im Frühjahr 1939 stellte Broch diesen 'Vorschlag' fertig. Es handelte sich dabei um die Ausarbeitung einer Idee, deren Realisierung Broch erstmals in seiner 'Völkerbund-Resolution' von 1937 vorgeschlagen hatte. Er schickte diese Projektstudie u. a. an das Institute for Advanced Study, Princeton, zu Händen von Albert Einstein und an Alvin Johnson, Direktor der New School for Social Research, New York City. Beide ermunterten Broch, den in der Studie umrissenen Fragestellungen selbst nachzugehen. Einstein sprach sich gegen eine besondere Institutsgründung aus, aber er vermittelte in den folgenden Monaten Brochs Kontakt zum Office of Public Opinion Research der Princeton University."

Oesers Exemplar, nach dem hier zitiert wird, umfaßt 23 Seiten, dazu das im Brief vom 30. 7. 1939 erwähnte Einlageblatt 4A, das zwar nach dem Erhalt von Oesers Brief versendet wurde, aber vermutlich schon vorher fertig war und jedenfalls von dessen Kritik offenbar unbeeinflußt ist.

13 Eric Herd im Vorwort zu den Briefen an Willa Muir in:

Gesammelte Werke, Bd. 10, Zürich 1961, S. 313.

14 Vgl. Gesammelte Werke, Bd. 10: Die Briefe an Willa Muir. Hrsg. von Eric Herd, Zürich 1961, S. 369.

15 Vgl. Gesammelte Werke, Bd. 9: Massenpsychologie, Zürich 1959, S. 15 und $77 \mathrm{ff}$.

16 Vgl. z. B. die Rezension des Bandes "Massenwahntheorie" der Kommentierten Werkausgabe, Frankfurt a.M. 1979, Literatur und Kritik, Nr. 138 (September 1979), S. 500 - 501 (Klaus Amman). 